\title{
Development of modify random waypoint mobility model of routing protocol for the mobile ad-hoc network
}

\author{
${ }^{1 *}$ Margam Suthar, ${ }^{2}$ Ajay Kumar Vyas Ph.D, SMIEEE \\ ${ }^{1}$ Research Scholar, Department of Electronics \& Communication Engineering, Gujarat Technological \\ University, Ahmedabad, Gujrat, India \\ ${ }^{2}$ Assitant Professor, Adani Institute of Infrastructure Engg, Gujarat Technological University, Ahmedabad, \\ Gujrat, India \\ *Corresponding author E-Mail Id: apl_mcwt@gtu.edu.in,_Orcid ID 0000-0003-2306-3832 \\ Ajay_ap7@yahoo.com
}

\begin{abstract}
Mobility models are used to evaluated the network protocols of the ad hoc network using the simulation. The random waypoint model is a model for mobility which is usually used for performance evaluation of ad-hoc mobile network. Mobile nodes have the dynamic mobility in the ad hoc network so the mobility model plays an important role to evaluate the protocol performance.

In this article, we developed modify random waypoint mobility (MRWM) model based on random waypoint for the mobile ad hoc network. In this article, the comparative analysis of modifying random waypoint mobility and random waypoint model on the ad hoc On-Demand Distance Vector (AODV) routing protocol has been done for large wireless ad hoc network (100 nodes) with the random mobile environment for the $1800 \mathrm{~s}$ simulation time. To enhance the confidence on the protocol widespread simulations were accomplished under heavy traffic (i.e. 80 nodes) condition. The proposed model protocol has been investigated with the performance metrics: throughput; packet delivery ratio; packet dropping ratio; the end to end delay and normalized routing overhead. The obtained results revealed that proposed modify random waypoint mobility model reduces the mobility as compared to the random waypoint mobility model and it is trace is more realist.
\end{abstract}

Keyword: Protocol; Mobile ad hoc network; Mobility Model; Random Waypoint Mobility; throughput.

\section{Introduction}

Mobile communication is an increasingly imperative part of every business [1]. To optimize the performance of mobile network different networks structure are suggested. The mobile ad hoc network is the multi-hop mobile node communication in the wireless environment without having any static arrangement [2]. A node in a wireless network is moveable, and its movement is random in nature. Thus it is complicated to develop the protocol for such an environment. The wireless network can be established in any structure and nodes are able to communicate within the transmission range. If the destination node within the range of the source node then the receiver node directly received the data otherwise sender node transmits the data through the intermediate node. Due to this reason, routing is required in the wireless network [3-4] with dynamic network topology [5]. The traditional protocol designed for the fixed infrastructure network so that is not suitable to use for the MANET network. In such a dynamic environment of the node, it is difficult to develop routing protocol that able to offer serves like routing, 
quality of service, resource management, network operation, and management for the mobile network. A routing protocol for such a dynamic wireless network can be design by considering the speed and mobility pattern of the node in the network. Thus it is very important to identify and trace of the node mobility pattern and its effect on the network as well as the protocol performance.

Different models are suggested so far to verify the various protocols. In which, a random waypoint model is one of the most preferable among those models. Some of the models are based on the mitigating black hole effects through detection and prevention (MBDP-AODV) based on a dynamic threshold value of the destination sequence number [6], another model for improve the traffic load in the network and identify a short and secure route detect a number of malicious nodes [7]. This model described the black hole detection using dynamic source routing (DSR) algorithm for a small network (i.e. 30 nodes) [8], another model is based on a trust-aware scheme to detect the intrusion in the MANET to improve the trust factors along with the direct trust, indirect trust, and the recent trust [9] and enhanced random mobility model, which belongs to the class of random direction models in which the movement trace of individual mobile is considered for short simulation time (i.e. 300sec)[10].

It is difficult to obtain real-life mobility patterns or trace-based mobility models [11] to analyze the wireless ad hoc network. In this study, we have examined that effect of random mobility (or trace-based mobility models) and random waypoint mobility model on routing protocol in MANET.

In this article, section 2 provides detail about the mobility model, section 3 described the comparative study of the random waypoint model and proposed model, section 4 discussed the all obtained results and section 5 present the conclusion of the proposed work.

\section{Mobility Model}

Mobile ad hoc network node has the random mobility, so to check the performance of the protocol, it is required to develop mobility model that defined the movement of the node, velocity or it describes the characteristic of the node. There are numerous protocols that are proposed by the researcher and protocol have the particular advantages and disadvantages based on the network scenario. Consequently, it is very imperative that select the protocol based on the mobility pattern. To represent the wireless mobility researcher proposed many mobility models such as random walk mobility model [12], random waypoint mobility model[13], random direction mobility model [14], boundless simulation area mobility model [15], Gauss-Markov mobility model [16], probabilistic version of the random walk mobility model [17] and City Section Mobility Model [18].

It broadly classified into two categories: trace and syntactic. Real-life mobility pattern of the node created in trace-based mobility model [19]. But to develop such mobility model and generate mobility trace become the major challenges for long observation time and the large mobile ad hoc network [20]. The various models are already proposed to represent the real-life realistic mobility pattern and characteristic[21]. Recent work evaluates the protocol performance based on the syntactic mobility model [22].

Individual mobile movement and group mobile movement are the two type of the systematic mobility model [23]. Mobility model characterizes the node mobility pattern in the network with respect to time in different network circumstances and other mobility model developed that represents the group of the node traversing pattern. Node moves randomly in the network so in syntactic mobility considered the randomness of the node and based on the randomness it classified into constrained topology-based models and statistical models. Constrained topology models consider partial randomness and in statistical model consider the randomness or the nodes. It allowed to node moved randomly anywhere in the network with the properly 
defined speed and direction [24]. Based on the features mobility models classified into four types as shown in figure 1:

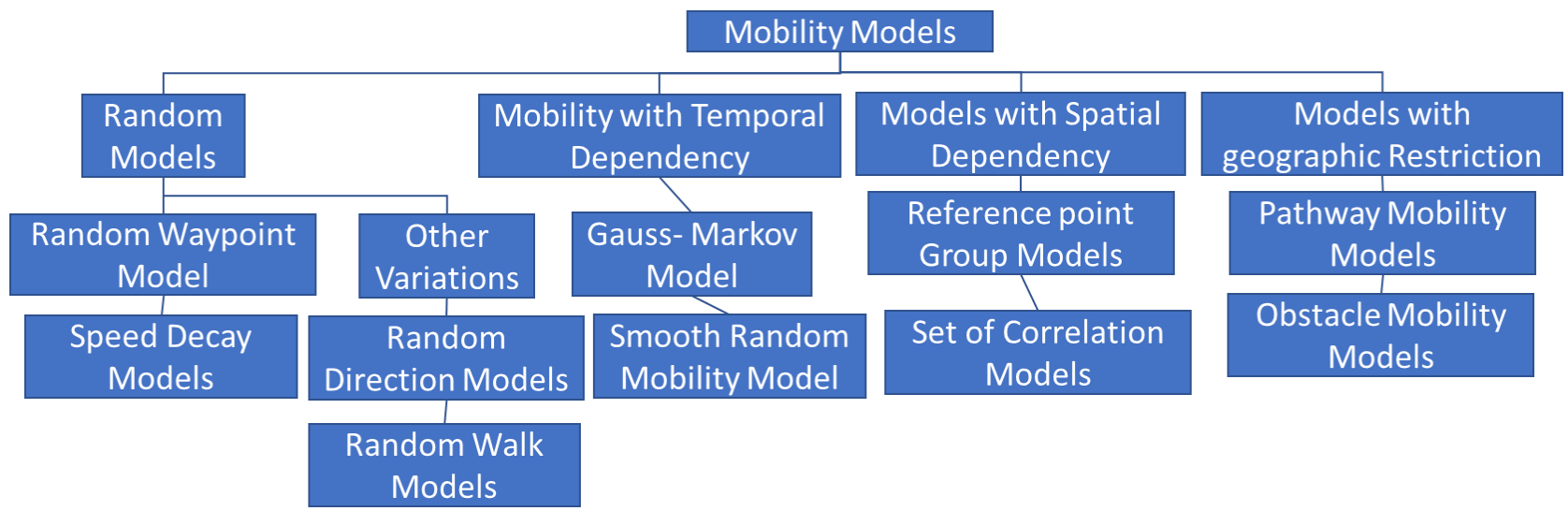

Figure 1: Classification of Mobility Model on the basis of the random models, mobility with temporal dependency, models with spatial dependency and model with geographic restraction

Random Models: Node moves randomly like the statistical model and based on the randomness properties it further classified into waypoint, random direction and random walk mobility.

The model with Temporal Dependency: In this model, Based on the effect of the node movement history mobility model patter decided. It further classified into Gauss-Markov and smooth random mobility model.

The model with Spatial Dependency: In this mobile model, mobile node travel in an interrelated manner. Example: Reference point group mobility model and Spatially correlated mobility models.

Models with Geographic restriction: Based on obstacles, streets and freeways mobility model developed. Example: pathway mobility model and obstacle mobility model.

\section{Random Model}

In the simulation-based evaluation of the wireless network, it is a very important role in the mobility model. Based on the different network protocol and scenario researcher chose the different mobility model for the protocol simulation [25,26,27,28]. Random mobility model is more commonly and frequently used by the researcher for the evaluation of the ad hoc protocol performance using the NS2[29] and GloMoSim[30] simulator[31,32]. In this article, we have done a comparative analysis of AODV [33] routing protocol performance in the random waypoint mobility model and the proposed mobility model.

\subsection{Random Waypoint Mobility Model:}

In this model node velocity, time and random destination point $(\mathrm{x}, \mathrm{y})$ coordinate decided randomly when node reach at the destination also it pause for some amount of time at the destination. Pause time at destination will be decided according to the more random variable after the pause time expire it repeat the node mobility movement and it decides the new velocity, pause time and destination coordinate. Similarly, it repeats the process. Starting and destination position defined as the waypoint and node travel distance between the starting to the destination waypoint is called the transition length 


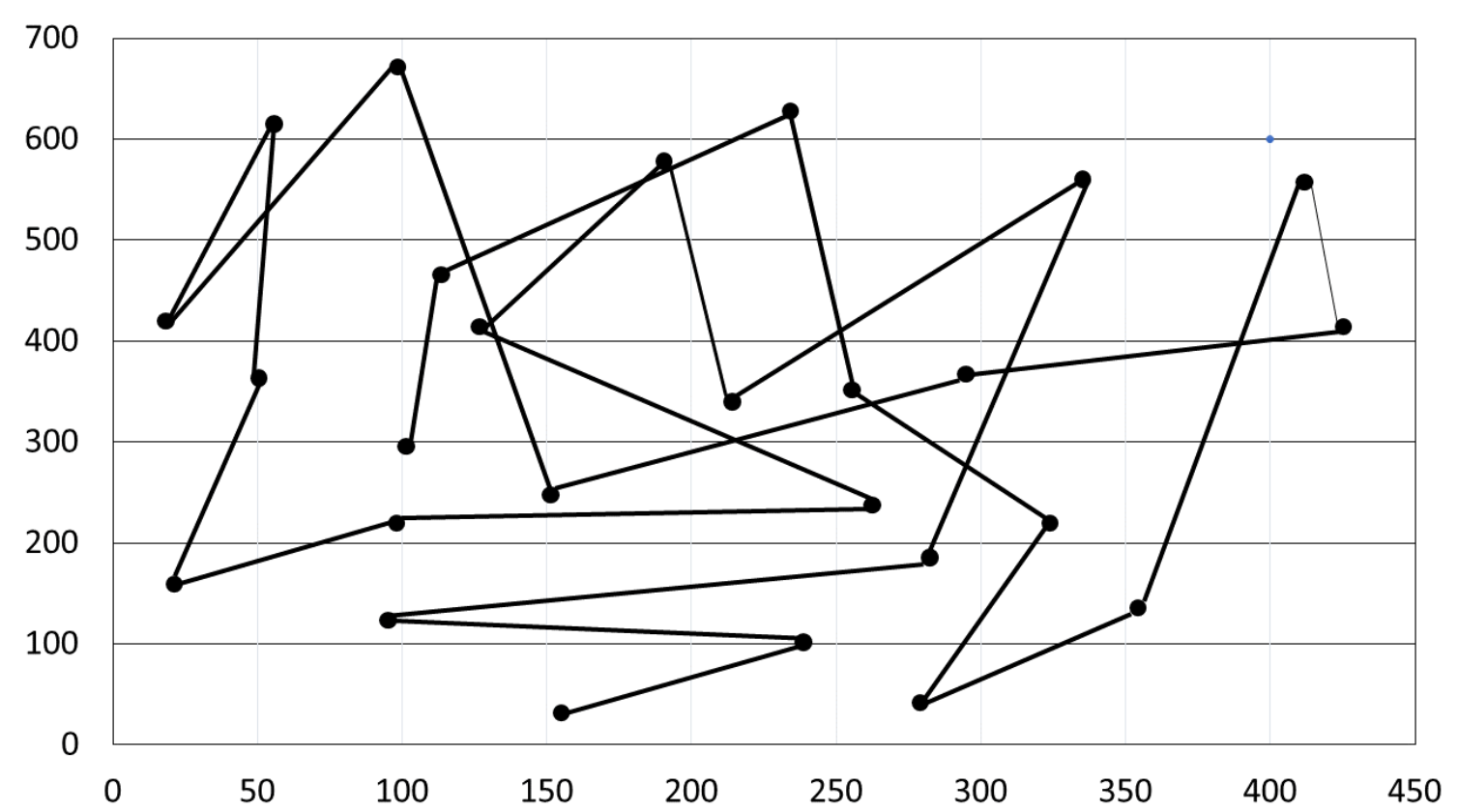

Figure 2:Random waypoint mobility model traveling pattern of a mobile node in random direction with variable speed.

Figure 2. shows the example of the random waypoint mobility model. In the article, we investigated initially ad-hoc network all the node randomly distributed in the simulation area. Initial randomly chouse the node coordinate $(133,180)$, speed of the node defined between the 0 to $10 \mathrm{~m} / \mathrm{s}$ and pause time decided to zero.

To capture and quantify the model speed notion, Johansson, Larsson and Hedman et al.[34] proposed the mobility metric to measure the relative speed between the node I and $\mathrm{j}$ at time $\mathrm{t}$ is given in equation (1)

$$
R S(i, j . t)=\left|\overrightarrow{V_{l}(t)}-\overrightarrow{V_{J}(t)} / M\right|
$$

The mobility metric $\bar{M}$ is calculated as the measure of relative speed averaged over all node pairs and over all time. It can be defined as eqn (2)

$$
\bar{M}=\frac{1}{|i . j|} \sum_{i=1}^{N} \sum_{j=i+1}^{N} \frac{1}{T} \int_{0}^{T} R S(i, j, t) d t
$$

Where $|i . j|$ is the number of distinct node pair $(i, j), n$ is the total number of node in the simulation field (i.e., ad hoc network), and $\mathrm{T}$ is the simulation time.

CMU Monarch group added the extension in the NS2 to create the wired as well as wireless simulation environment [35]. For the evaluate the routing protocol performance, "set dest" tool included in the Network Simulation (ns 2) to generate the Random Waypoint model for the routing protocol.

\subsection{Modify random waypoint mobility (MRWM) Model}

In real life, ad hoc network node move randomly and due to this the topology of the network change dynamically. So in the proposed random mobility model having node move randomly without any restriction in the network. Also node mobility direction, Speed and destination 
point $(\mathrm{x}, \mathrm{y})$ very dynamically. To analyze routing protocol performance, we generate dynamic random mobility in network simulator:

\$ set mobility [lindex \$argv 0]; \# Dynamic mobility file. This is used for random mobility using for loop which is not used in Random waypoint Model.

Node move randomly in the network and its location speed and direction vary with respect to the time. So, in this analysis, for the same scenario we generate result for ten time and take average result of them.

\section{Experiment Work:}

Comparative analysis for AODV routing protocol performance in the modify random waypoint mobility (MRWM) and random mobility model has been carried out using the Network Simulator 2 (NS2). The parameters specification for proposed work is given in table1.

Table 1:Parameters Specification is choose for the experiments

\begin{tabular}{|l|l|}
\hline Parameter & Typical Value \\
\hline Simulator Version & NS-2.35 \\
\hline Routing Protocols & AODV \\
\hline Network Space & $600 \mathrm{~m}$ x 600 m \\
\hline No. of nodes & 100 \\
\hline Mobility Model & $\begin{array}{l}\text { Modify Random Waypoint Mobility } \\
\text { (MRWM) and Random Waypoint Mobility }\end{array}$ \\
\hline Speed & Min 1m/s to Max 10m/s \\
\hline Traffic Type & UDP \\
\hline MAC Protocol & IEEE 802.11 \\
\hline Simulation Time & $1800 \mathrm{~s}$ \\
\hline Packet Size & 512 \\
\hline
\end{tabular}

In the comparative analysis, we have used the ad hoc network having the 100 mobile nodes and AODV routing protocol for the data transmission in the network. To check the effect of the mobility on the protocol, we increase the traffic in the network. Initially, we analyse AODV protocol performance in the network by using 100 number of node and 10 communicating nodes for the simulation time of the $1800 \mathrm{sec}$ after that we increase the traffic in the network by the 10 up to the 80 communicating nodes. In the proposed work, we consider random mobility, so the node moves randomly in the network with the velocity of $10 \mathrm{~m} / \mathrm{s}$. The results carried out by considering the average of the 10 times pattern for each set of the node.

\section{Results Analysis}

For the comparative analysis has been of proposed MRWM with random mobility on network protocol performance is carried out with four performance parameters as discussed below:

Throughput: It measures the ratio of the total number of the data received at the receiver from the sender to the time taken by the receiver to receive all the data. 


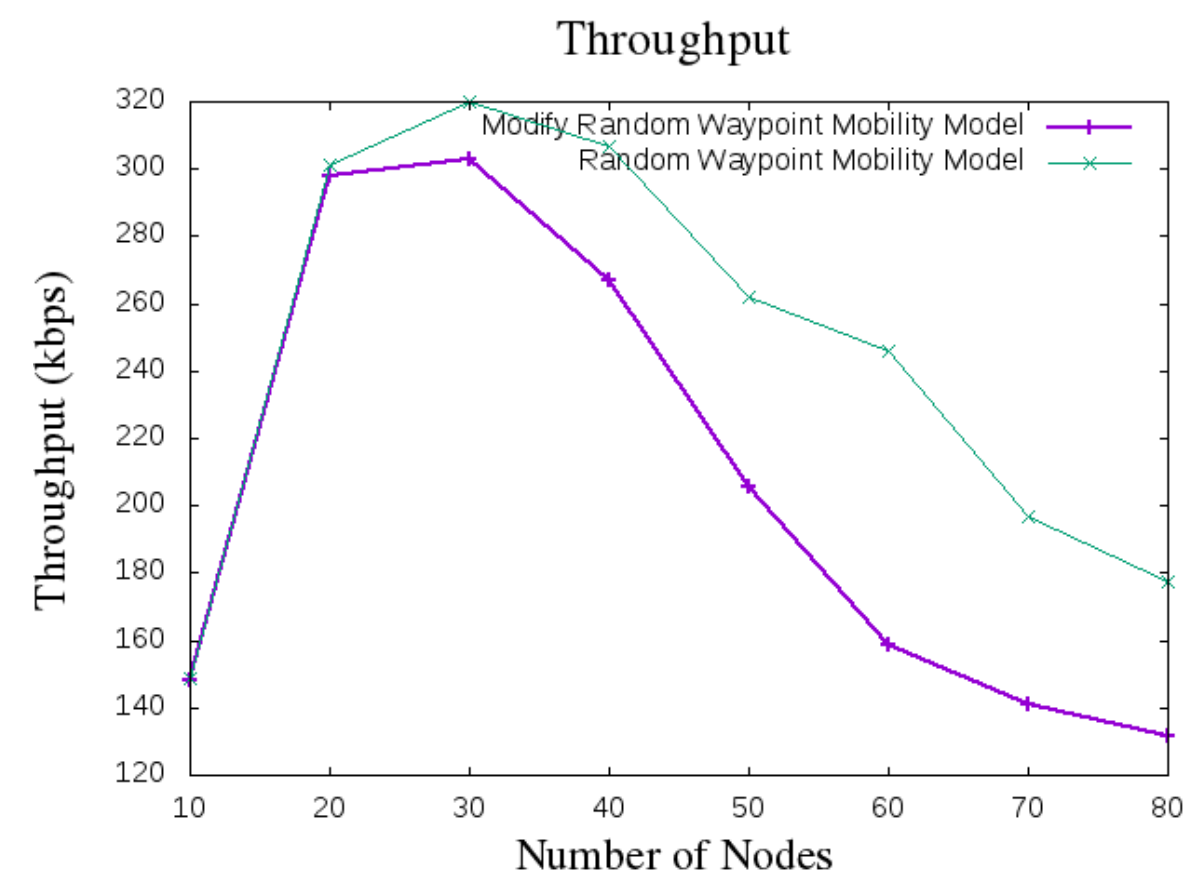

Figure 3: Throughput verses number of nodes for the proposed model(purple color with dash marker) and random waypoint model (green color with cross marker) having similar throghtput upto 20 nodes.

Table 2: Throughput analysis of proposed model with RWP for different nodes

\begin{tabular}{|c|c|c|}
\hline \multicolumn{2}{|c|}{ Throughput } \\
\hline Number of node & $\begin{array}{c}\text { Modify random waypoint } \\
\text { mobility (MRWM) }\end{array}$ & $\begin{array}{c}\text { Random Waypoint Mobility } \\
\text { (RWP) }\end{array}$ \\
\hline 10 & 147.952 & 148.52 \\
\hline 20 & 297.709 & 301.46 \\
\hline 30 & 302.650 & 320.00 \\
\hline 40 & 267.003 & 306.61 \\
\hline 50 & 205.784 & 261.84 \\
\hline 60 & 158.587 & 245.89 \\
\hline 70 & 140.816 & 196.91 \\
\hline 80 & 131.620 & 177.31 \\
\hline
\end{tabular}

Figure 3 shows the AODV protocol throughput analysis in the Modify random waypoint mobility (MRWM) and random waypoint mobility model. The value of the throughput for different nodes are given in table 2 .

In the modify random waypoint mobility (MRWM) node mobility more dynamic compare to the random waypoint mobility So as the number of the connection (10 to 80 communicating node)increase in the network effect of the dynamic mobility also increase and it drops more number of the packet in the network compare the random waypoint mobility. At 10 communicating node, the modify random waypoint mobility (MRWM) throughput is 147.952 kbps and random waypoint mobility (RWP) throughput is $148.52 \mathrm{kbps}$. As the communicating node increase to 80, the throughput of PRM is $131.620 \mathrm{kbps}$ and RWP is 177.31 . 
Normalized Routing Overhead: It is defined as the total number of routing packets to the total number of delivered data packets at the destinations. Normalized routing overhead for both models at the different number of nodes is shown in figure 4 . The obtained values of the normalized routing overhead are given in table3.

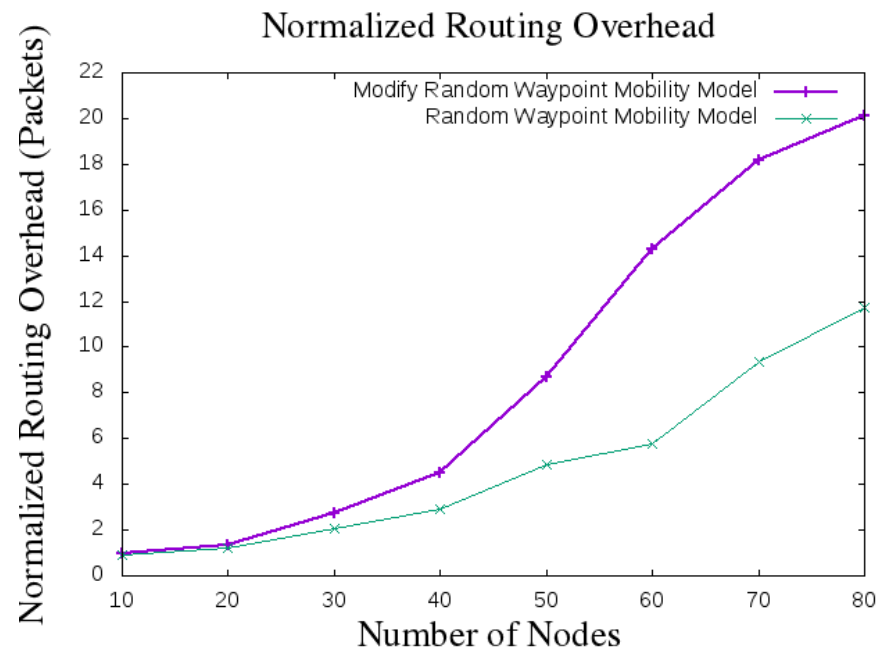

Figure 4: Normalized Routing overhead for the modify random waypoint mobility model(purple color with dash marker) and random waypoint model(green color with cross marker) having similar throghtput upto 20 nodes after 20 nodes the MRWMM maximum overhead packets.

Table 3:Normalized Routing Overhead

\begin{tabular}{|c|c|c|}
\hline \multicolumn{3}{|c|}{ Normalized Routing Overhead } \\
\hline Number of node & $\begin{array}{c}\text { Modify random waypoint } \\
\text { mobility (MRWM) }\end{array}$ & $\begin{array}{c}\text { Random Waypoint Mobility } \\
\text { (RWP) }\end{array}$ \\
\hline 10 & 0.9964 & 0.882 \\
\hline 20 & 1.3501 & 1.205 \\
\hline 30 & 2.7648 & 2.052 \\
\hline 40 & 4.4805 & 2.931 \\
\hline 50 & 8.6825 & 4.856 \\
\hline 60 & 14.2796 & 5.766 \\
\hline 70 & 18.1753 & 9.364 \\
\hline 80 & 20.1124 & 11.718 \\
\hline
\end{tabular}

In proposed MRWM node destination coordinate (x.y), speed and pause time very dynamically compare to the random waypoint mobility and we have take average of 10-time result for each set of node. Due to this, it is more difficult to maintain the routing path in the network and the more number of the routing packet required compare to the data delivered at the destination node. So the normalized routing overhead in the modify random waypoint mobility (MRWM) is higher or increase as the traffic increase form 10 to 80 node in the network. As traffic increase from 10 to 80 nodes the normalized routing overhead of MRWM and RWP increase from 0.996 to 20.112 and 0882 to 11.718 respectively in the 100 nodes network. 
Packet Dropping Ratio: It is defined as the total number of packet loss divide by the total number of packets sent.

In dynamic node mobility or modify random waypoint mobility (MRWM) model have unpredictable node movement, velocity and node coordinate $(\mathrm{x}, \mathrm{y})$ in the network compared to the random waypoint model. The packet drop ratio for MRWM and RWM is shoen in the figure 5 , it is revealed that the more number of packet drop in the modify random waypoint mobility (MRWM) model compare to the random waypoint mobility at 10 nodes the packet dropping ratio of the MRWM and RWP is 0.964 and 0.63 respectively. All other value for different number of nodes are given in table 4.

As the traffic increase in the network the packer dropping ratio also increase in the MRWM compare to the RWP model. MRWM and RWP packet dropping ratio at 80 nodes are 89.03 and 81.14 respectively.

\section{Packet Dropping Ratio}

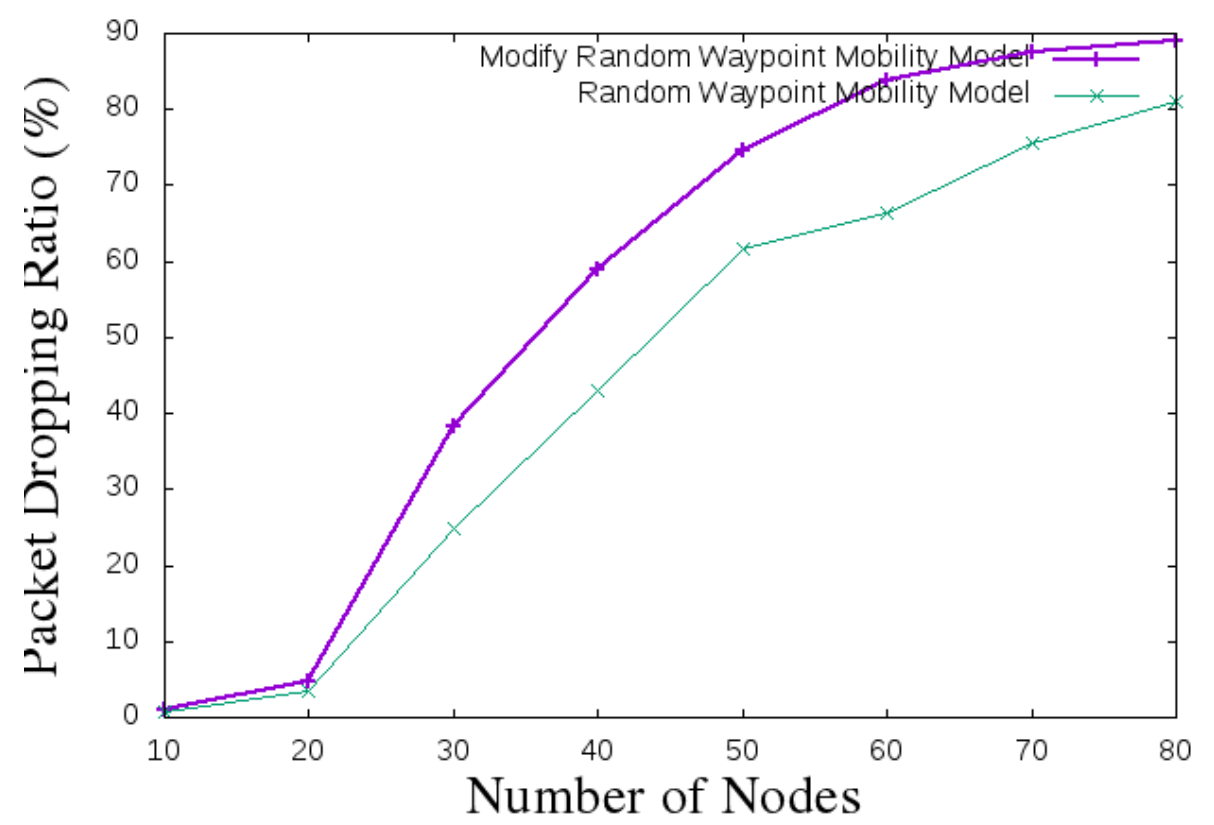

Figure 5: Packet Dropping Ratio for the modify random waypoint mobility model(purple color with dash marker) and random waypoint model(green color with cross marker) verses number of nodes.

Table 4:Packet Dropping Ratio

\begin{tabular}{|c|c|c|}
\hline \multicolumn{2}{|c|}{ Packet Dropping Ratio } \\
\hline Number of node & $\begin{array}{c}\text { Modify random waypoint } \\
\text { mobility (MRWM) }\end{array}$ & $\begin{array}{c}\text { Random Waypoint Mobility } \\
\text { (RWP) }\end{array}$ \\
\hline 10 & 0.964 & 0.63 \\
\hline 20 & 4.747 & 3.53 \\
\hline 30 & 38.259 & 24.84 \\
\hline 40 & 58.907 & 43.00 \\
\hline 50 & 74.584 & 61.64 \\
\hline 60 & 83.699 & 66.27 \\
\hline 70 & 87.585 & 75.69 \\
\hline 80 & 89.03 & 81.14 \\
\hline
\end{tabular}


Packet Delivery Ratio: it is defined as the total number of packets successfully received by total number of packets sent.

We analyzed the network performance in the network space of $600 \mathrm{~m}$ x $600 \mathrm{~m}$. As shown in figure 6, during the less traffic (10 nodes) performance of the AODV protocol more stable compare to the higher traffic. The value of the packet delivery ratio for network space is given in table 5. Now, as traffic increase within the network space $600 \mathrm{~m}$ to $600 \mathrm{~m}$, it will be dropping more number of the packet. As node increase from 10 to 80 the packet delivery ratio of the modify random waypoint mobility (MRWM) and random waypoint model is also decrease from 99.036 to 10.97 and 99.37 to 18.86 respectively.

\section{Packet Delivery Ratio}

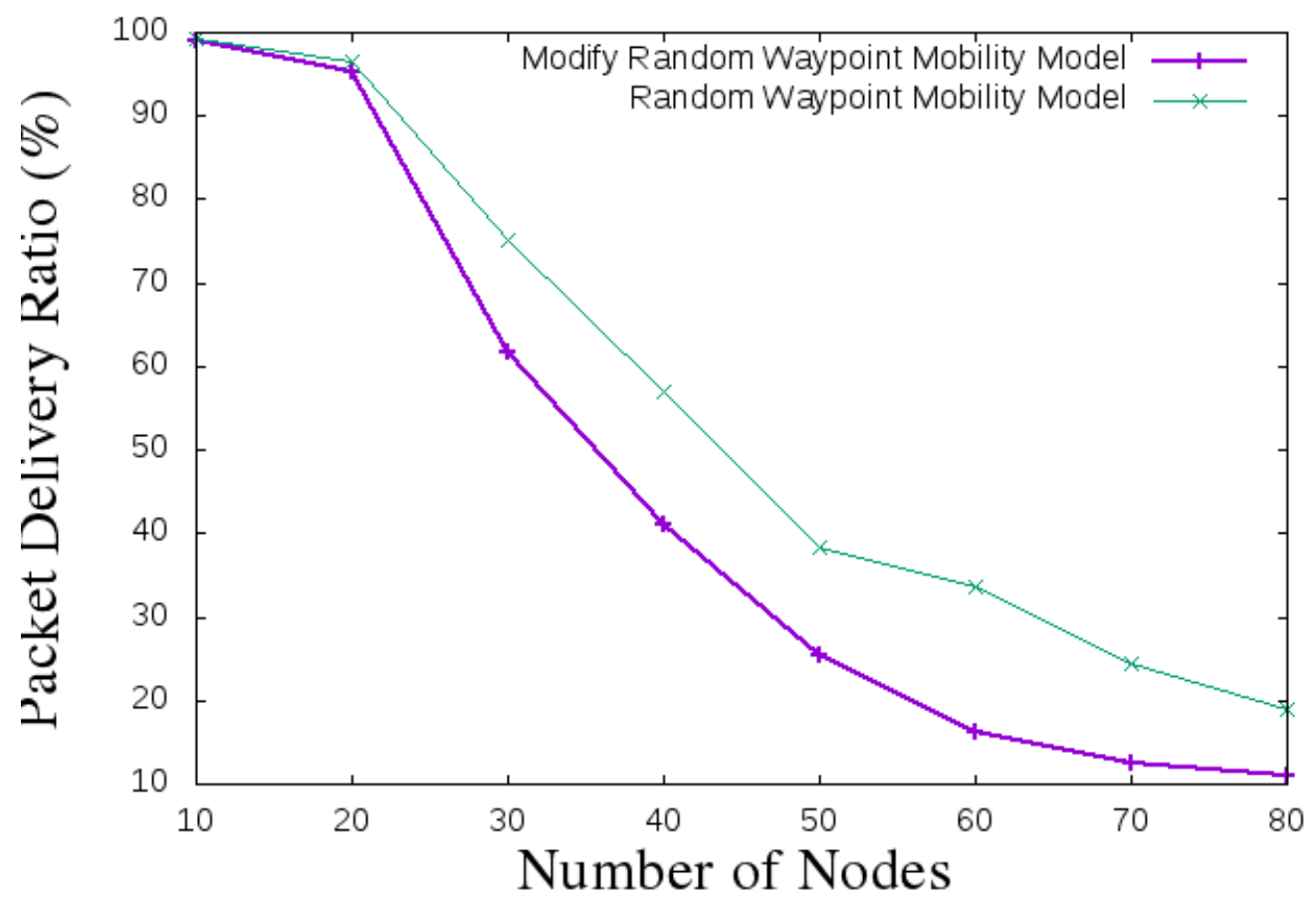

Figure 6: Racket Delivery Ratio for the modify random waypoint mobility model(purple color with dash marker) and random waypoint model (green color with cross marker) having similar ratio of less number of nodes but vary for greater number of nodes. .

Table 5: Packet Delivery Ratio

\begin{tabular}{|c|c|c|}
\hline \multicolumn{3}{|c|}{ Packet Delivery Ratio } \\
\hline Number of node & MRWM & RWP \\
\hline 10 & 99.036 & 99.37 \\
\hline 20 & 95.253 & 96.47 \\
\hline 30 & 61.741 & 75.16 \\
\hline 40 & 41.093 & 57.00 \\
\hline 50 & 25.416 & 38.36 \\
\hline 60 & 16.301 & 33.73 \\
\hline 70 & 12.415 & 24.31 \\
\hline 80 & 10.97 & 18.86 \\
\hline
\end{tabular}


End-to-End Delay: Average time required by all the data to deliver to their destination.

As traffic increase in 100 node large network, required end to end dealy in modify random waypoint mobility (MRWM) is highere compare to the randam waypoint model. Due to the dynaminc mobility the Modify random waypoint mobility (MRWM) model packet routing pathe change more frequently compare to randam waypoint model. So it increase the delay in the network to deliver the packet at the destination. From the figure 7 and table 6 , it conclude that the as traffic increase (10 to 10 nodes) in the network the end to end delay of Modify random waypoint mobility (MRWM) and random waypoint model also increase from 0.044 to 7.844 and 0.04 to 7.53 respectvely. At 80 traffice nodes dealy in MRWM (7.844) model is larger at compare to the RWP (7.53).

\section{End to end delay}

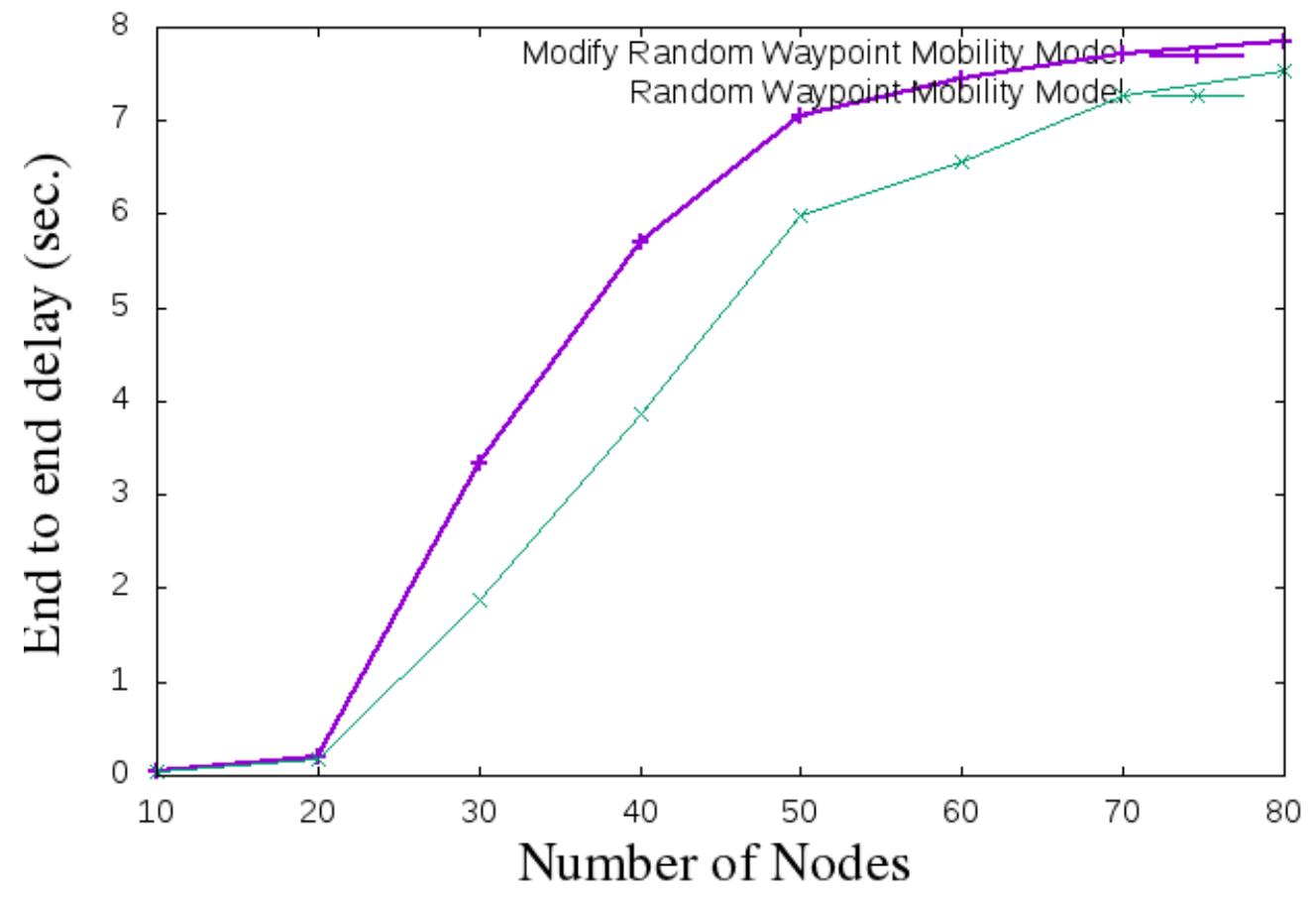

Figure 7:End to End Delay the modify random waypoint mobility model(purple color with dash marker) and random waypoint model(green color with cross marker)

Table 6: End to End Delay

\begin{tabular}{|c|c|c|}
\hline \multicolumn{2}{|c|}{ End to End Delay } \\
\hline Number of node & $\begin{array}{c}\text { Modify random waypoint } \\
\text { mobility (MRWM) }\end{array}$ & $\begin{array}{c}\text { Random Waypoint Mobility } \\
\text { (RWP) }\end{array}$ \\
\hline 10 & 0.044 & 0.04 \\
\hline 20 & 0.198 & 0.17 \\
\hline 30 & 3.344 & 1.88 \\
\hline 40 & 5.705 & 3.87 \\
\hline 50 & 7.048 & 5.98 \\
\hline 60 & 7.450 & 6.56 \\
\hline 70 & 7.720 & 7.27 \\
\hline 80 & 7.844 & 7.53 \\
\hline
\end{tabular}




\section{Conclusion}

In this study, we proposed Modify Random Waypoint Mobility (MRWM) model. The comparative analysis of the proposed model with random waypoint mobility (RWP) environment has been carried out on AODV protocol performance for the large mobile ad-hoc network (i.e.100 nodes) with the traffic increase in the network up to 80 nodes. Effect of the MRWM and RWP environment on AODV protocol has been analyzed with the performance metrics such as throughput, packet delivery ratio, packet dropping ratio, end to end delay and normalized routing overhead. As traffic increase from 10 to 80 nodes with 100 mobile nodes the AODV protocol throughput increase from 147.952 to $131.620 \mathrm{bits} / \mathrm{sec}$ and 148.52 to 177.31 bits/sec in MRWM and RWP respectively. Due to the dynamic mobility more, packet drop and less number of the packet deliver in MRWM compare to RWP. As more packet drop, to maintain the routing path protocol required to send more number of routing packet in the network so at 80 nodes traffic the NRO in 20.1124 \& 11.718 in MRWM \& RWP respectively. Also, increase the end to end delay in the network. From the comparative result, it concluded that the MRWM model mobility patter more real-life mobility pattern compare to the RWP mobility model.

\section{Acknowledgment:}

The authors are thankful to Dr Pranav B. Lapsiwala Sarvajanik College of Engg. \& Tech. Surat, Gujarat and Prof. Hitesh Shah GCET, Vallabh Vidyanagar (Gujarat) valuable discussion for research work.

Funding: No funding is received for the research work.

\section{Conflicts of interest: None}

\section{Reference}

[1] Libert, Barry, Megan Beck, and Jerry Wind. The network imperative: How to survive and grow in the age of digital business models. Harvard Business Review Press, 2016.

[2] S. Misra, I.Woungang, and S. C. Misra in (2009), Guide to Wireless Ad hoc Networks, Verlag London, UK, Computer Communication and networks Series, Springer.

[3] Boukerche, A., Turgut, B., Aydin, N., Ahmad, M. Z., Bölöni, L., \& Turgut (2011). Routing protocols in ad hoc networks: A survey. Elsevier Computer Networks Journal, Volume 55, Issue 13, 15, Pages 3032-3080, September 2011.

[4] Shahzana Memon, Pardeep Kumar, Umair Ali Khan, Tanesh Kumar (2015). Performance Evaluation of Mobile Ad Hoc Routing Mechanisms," Springer Wireless Personal Communications, Vols. Volume 85, Issue 2, p. pp 377-392.

[5] Shashi Gurung \& Siddhartha Chauhan. (2018). A dynamic threshold based approach for mitigating black-hole attack in MANET. Spinger Journal-Wireless Networks.

[6] T. Camp, J. Boleng, and V. Davies (2002). A survey of mobility models for ad hoc network research. Wireless Comm. and Mobile Computing (WCMC), 2(5):483-502.

[7] Taher Delkesh \& Mohammad Ali Jabraeil Jamali. (2019). EAODV: detection and removal of multiple black hole attacks through sending forged packets in MANETs. Journal of Ambient Intelligence and Humanized Computing volume 10, pages18971914. 
[8] Peter Ndajah, Abdoul Ousmane Matine \& Mahouton Norbert Hounkonnou (2019). Black Hole Attack Prevention in Wireless Peer-to-Peer Networks: A New Strategy. International Journal of Wireless Information Networks volume 26, pages48-60.

[9] Neenavath Veeraiah \& B. Tirumala Krishna (2019). Trust-aware Fuzzy Clus-Fuzzy NB: intrusion detection scheme based on fuzzy clustering and Bayesian rule. Wireless Networks volume 25, pages4021-4035.

[10] C. Bettstetter (2001). Smooth is better than sharp: A random mobility model for simulation of wireless networks. In Proceedings of MSWiM'01. ACM.

[11] Aschenbruck, Nils, Aarti Munjal, and Tracy Camp. "Trace-based mobility modeling for multi-hop wireless networks." Computer Communications 34, no. 6 (2011): 704714

[12] Chiang, Kuo-Hsing, and Nirmala Shenoy,A 2-D random-walk mobility model for location-management studies in wireless networks. IEEE Transactions on vehicular technology 53, no. 2 (2004): 413-424.

[13] Hernández-Orallo, Enrique, and Antonio Armero-Martínez,How Human Mobility Models Can Help to Deal with COVID-19.Electronics 10, no. 1 (2021): 33.

[14] Carofiglio, Giovanna, Carla-Fabiana Chiasserini, Michele Garetto, and Emilio Leonardi.Route stability in MANETs under the random direction mobility model, IEEE transactions on Mobile Computing 8, no. 9 (2009): 1167-1179.

[15] Roy, Radhika Ranjan,Boundless simulation area mobility, In Handbook of Mobile Ad Hoc Networks for Mobility Models, pp. 379-403. Springer, Boston, MA, 2011.

[16] Meghanathan, Natarajan, Impact of the Gauss-Markov mobility model on network connectivity, lifetime and hop count of routes for mobile ad hoc networks, Journal of networks 5, no. 5 (2010): 509.

[17] Gupta, Anuj K., Harsh Sadawarti, and Anil K. Verma, Performance analysis of MANET routing protocols in different mobility models, International Journal of Information Technology and Computer Science (IJITCS) 5, no. 6 (2013): 73-82.

[18] Hossain, Md Shohrab, and Mohammed Atiquzzaman, Stochastic properties and application of city section mobility model. In GLOBECOM 2009-2009 IEEE Global Telecommunications Conference, pp. 1-6. IEEE, 2009.

[19] Casella, Enrico, Marco Ortolani, Simone Silvestri, and Sajal K. Das. Hierarchical syntactic models for human activity recognition through mobility traces. Personal and Ubiquitous Computing 24, no. 4 (2020): 451-464.

[20] Zheng, Q et al (2004). Recent Advances in Mobility Modeling for Mobile Ad Hoc Network Research. Recent advances in mobility modeling for mobile ad hoc network research. Pages 70-75 https://doi.org/10.1145/986537.986554.

[21] Bettstetter, Christian. Mobility modeling in wireless networks: categorization, smooth movement, and border effects, ACM SIGMOBILE Mobile Computing and Communications Review 5, no. 3 (2001): 55-66.

[22] Royer, Elizabeth M., and Chai-Keong Toh., A review of current routing protocols for ad hoc mobile wireless networks, IEEE personal communications 6, no. 2 (1999): 4655.

[23] Bai, F., Sadagopan, N., \& Helmy, A. (2003, March). IMPORTANT: A framework to systematically analyze the Impact of Mobility on Performance of RouTing protocols for Adhoc NeTworks. In IEEE INFOCOM 2003. Twenty-second Annual Joint 
Conference of the IEEE Computer and Communications Societies (IEEE Cat. No. 03CH37428) (Vol. 2, pp. 825-835). IEEE.

[24] Jardosh, Amit, Elizabeth M. Belding-Royer, Kevin C. Almeroth, and Subhash Suri. Towards realistic mobility models for mobile ad hoc networks. In Proceedings of the 9th annual international conference on Mobile computing and networking, pp. 217-229. 2003.

[25] Chlamtac, Imrich, Marco Conti, and Jennifer J-N. Liu. Mobile ad hoc networking: imperatives and challenges. Ad hoc networks 1, no. 1 (2003): 13-64.

[26] Manjeshwar, Arati, and Dharma P. Agrawal. TEEN: ARouting Protocol for Enhanced Efficiency in Wireless Sensor Networks. In ipdps, vol. 1, p. 189. 2001.

[27] Hong, M. Gerla, G. Pei and C.-C. Chiang (1999). A group mobility model for ad hoc wireless networks, in: Proc. ACM International Workshop on Modeling, Analysis, and Simulation of Wireless and Mobile Systems (MSWiM), Seattle, WA.

[28] J.G. Markoulidakis, G.L. Lyberopoulos, D.F. Tsirkas and E.D. Sykas (1997) Mobility modeling in third-generation mobile telecommunication systems, IEEE Personal Comm. 41-56.

[29] The network simulator - ns-2 (June 2002). http://www.isi.edu/nsnam/ns/

[30] Glomosim. (November 2002) http://pcl.cs.ucla.edu/projects/glomosim/ .

[31] S.R. Das, C.E. Perkins and E.M (2000). Royer, Performance comparison of two ondemand routing protocols for ad hoc networks, in: Proc. IEEE InfoCom, Tel Aviv, Israel.

[32] G. Holland and N.H. Vaidya. (1999). Analysis of TCP performance over mobile ad hoc networks, in: Proc. ACM International Conference on Mobile Computing and Networking (MobiCom), Seattle, WA.

[33] J. Broch, D. A. Maltz, D. B. Johnson, Y.-C. Hu, and J. Jetcheva (1988). A performance comparison of multi-hop wireless ad hoc network routing protocols. in Proceedings of the Fourth Annual ACM/IEEE International Conference on Mobile Computing and Networking(Mobicom98), ACM.

[34] P. Johansson, T. Larsson, N. Hedman, B. Mielczarek, and M. Degermark, Scenariobased performance analysis of routing protocols for mobile ad-hoc networks, in International Conference on Mobile Computing and Networking (MobiCom'99), 1999, pp. 195--206.

[35] CMU Monarch group, CMU Monarch extensions to the NS- 2 simulator (1988) http://monarch.cs.cmu.edu/cmu-ns.html 